\title{
Corticosteroids in the treatment of dengue shock syndrome
}

This article was published in the following Dove Press journal:

Infection and Drug Resistance

22 May 2014

Number of times this article has been viewed

\author{
Senaka Rajapakse' \\ Chaturaka Rodrigo' \\ Sachith Maduranga' \\ Anoja Chamarie Rajapakse ${ }^{2}$ \\ 'Department of Clinical Medicine, \\ Faculty of Medicine, University of \\ Colombo, Colombo, Sri Lanka; ${ }^{2}$ Kings \\ Mill Hospital, Sherwood Forest NHS \\ Foundation Trust, Sutton-in-Ashfield, \\ Nottinghamshire, UK
}

\begin{abstract}
Dengue infection causes significant morbidity and mortality in over 100 countries worldwide, and its incidence is on the rise. The pathophysiological basis for the development of severe dengue, characterized by plasma leakage and the "shock syndrome" are poorly understood. No specific treatment or vaccine is available, and careful monitoring and judicious administration of fluids forms the mainstay of management at present. It is postulated that vascular endothelial dysfunction, induced by cytokine and chemical mediators, is an important mechanism of plasma leakage. Although corticosteroids are potent modulators of the immune system, their role in pharmacological doses in modulating the purported immunological effects that take place in severe dengue has been a subject of controversy. The key evidence related to the role of corticosteroids for various manifestations of dengue are reviewed here. In summary, there is currently no high-quality evidence supporting the beneficial effects of corticosteroids for treatment of shock, prevention of serious complications, or increasing platelet counts. Non-randomized trials of corticosteroids given as rescue medication for severe shock have shown possible benefit. Nonetheless, the evidence base is small, and good-quality trials are lacking. We reiterate the need for well-designed and adequately powered randomized controlled trials of corticosteroids for the treatment of dengue shock.
\end{abstract}

Keywords: dengue, dengue shock, shock, corticosteroids, vascular leak, thrombocytopenia

\section{Dengue overview}

Dengue is a mosquito-borne acute febrile illness. It is caused by a flavivirus with four distinct serotypes (DENV-1, 2, 3, 4). ${ }^{1}$ Dengue is spread between humans by mosquitoes of the Aedes genus, ie, Aedes aegypti and Aedes albopictus. ${ }^{2}$ Following infection with one of the serotypes, lifelong immunity develops, which is type specific. Serious disease occurs frequently, though not exclusively, as a result of a second infection by a different serotype. ${ }^{3}$ The exact reasons or mechanisms that result in the development of the severe, life-threatening dengue shock syndrome remain an enigma. The principal pathophysiological phenomenon that occurs is acute vascular leakage, ${ }^{4}$ which lasts for 24-48 hours after its onset.

The incidence of dengue is rising. During the period 1955-1959, the average annual number of dengue infections reported to the World Health Organization (WHO) was just 908 from less than ten countries; this had risen to 925,896 from more than 60 countries during the period $2000-2007 .{ }^{5}$ It is currently estimated that 390 million infections occur annually, in over 100 countries; 96 million of these manifest clinically. ${ }^{6}$ Dengue epidemics follow seasonal climatic change; waves of epidemics occur during each rainy season. Thousands may be affected during epidemics. While most patients
Correspondence: Senaka Rajapakse Tropical Medicine Research Unit, Department of Clinical Medicine, Faculty of Medicine, University of Colombo, 25 Kynsey Road,

Colombo 08, Sri Lanka

Tel +94 II 2695300

Fax +94 II 2689188

Email senaka.ucfm@gmail.com;

senaka@tropmed.cmb.ac.lk 
recover from a simple febrile illness, a small but significant proportion go on to develop the dengue shock state, with associated fatalities. In affected areas, the case fatality rate of the more severe infections is $1 \%$ or higher, particularly in children and young adults. ${ }^{7}$ Traditionally thought to affect children, significant numbers of adults are also affected, ${ }^{8}$ resulting in considerable economic impact. The difficulty in controlling dengue infection stems from three root causes: non-availability of specific treatment, lack of an effective vaccine, and difficulties in vector control.

\section{Pathogenesis of dengue}

The pathogenesis of severe dengue is poorly understood. One factor that is thought to cause the dreaded shock syndrome is antibody-dependent enhancement, resulting in increased viral replication; ${ }^{3,9,10}$ however, many other virus and host factors are thought to contribute. ${ }^{1-13}$ Much of the evidence points to severe manifestations of dengue having an immunological basis, ${ }^{9,14-16}$ rather than being due to direct tissue damage by the virus. Variations in virulence in the infecting strain may contribute, and higher viral loads correlate with disease severity. ${ }^{17,18}$ Vascular endothelial cell dysfunction, induced by cytokine and chemical mediators, is thought to be an important factor leading to plasma leakage. Current limited evidence suggests that transient disruption of the surface glycocalyx lining the vascular endothelium takes place. ${ }^{19}$ The cytokines tumor necrosis factor alpha, interleukin (IL)-2, IL-6, IL-8, IL-10, IL-12, and interferon gamma are significantly elevated in severe dengue when compared with uncomplicated dengue fever. ${ }^{17}$ Complement activation is a feature of severe dengue, and complement levels correlate with disease severity.

Corticosteroids in high doses are potent modulators of the immune system and are of proven benefit in many conditions with deranged immunity. Their clinical use in septic or inflammatory shock has, however, been fraught with controversy. During the last 2 decades, studies showed much promise regarding their benefit in septic shock in patients with sepsis-induced adrenal suppression. ${ }^{20}$ However, this controversy is yet unresolved, and currently the guidelines for the treatment of severe sepsis recommend corticosteroids in low doses (ie, hydrocortisone $200 \mathrm{mg}$ daily by continuous infusion) only in patients with refractory shock, and, furthermore, do not recommend the differentiation between patients with and without an adequate adrenocortical response. ${ }^{21}$ Nonetheless, in sepsis, corticosteroids are generally safe, with hyperglycemia and hypernatremia the only clinically significant adverse effects. ${ }^{22}$ Though there have been concerns previously that corticosteroids may increase the incidence of superinfection and gastrointestinal bleeding, these are largely unsupported. ${ }^{22,23}$ The beneficial effects of low-dose corticosteroids in septic shock are presumed to be due to restoration of vascular reactivity to vasopressor agents and not their immunosuppressive effects. Lack of vascular reactivity to vasopressors is not considered to be the main mechanism of shock in dengue, and thus the evidence from severe sepsis cannot be directly extrapolated to dengue.

In acute respiratory distress syndrome, high-dose corticosteroids reduced the levels of cytokines tumor necrosis factor alpha, IL-1, IL-6, and IL-8; ${ }^{24}$ however, dexamethasone was shown to have no effect on reducing IL-8 levels in dengue. ${ }^{25}$ In another recent study, which was part of a randomized controlled trial conducted to determine the effects of high-dose corticosteroids given early in the course of dengue illness, there was no attenuation in the levels of acute phase plasma cytokine concentrations with the administration of corticosteroids. ${ }^{26}$ Interestingly, this study did not demonstrate markedly elevated levels of plasma cytokines in response to dengue, which is in contrast to the previous body of evidence.

\section{Clinical manifestations}

The natural history of dengue infection is fairly straightforward. The incubation period following inoculation of the virus is around 4-7 days. Beginning as an acute febrile illness, characterized by high temperature, malaise, retro-orbital headache, myalgia, backache, nausea, loss of appetite, and vomiting, the hallmark of severe dengue is capillary leakage, which occurs during the third to seventh day of illness. This coincides with reduction in viremia, ${ }^{27}$ suggesting its immunopathogenic basis. ${ }^{9,14}$ The symptomatic phase of dengue is, for management purposes, divided into three phases: the febrile phase, the critical phase, and the recovery phase. $^{5}$ During the febrile phase, patients have constitutional symptoms described above, but are generally hemodynamically stable; dehydration can occur due to severe vomiting. Platelet counts decline, leukopenia is a feature, and varying degrees of hemoconcentration can occur. Many patients make an uneventful recovery, with abatement of fever and constitutional symptoms, and the patient is then considered to have had simple dengue fever. Severe dengue occurs in a minority, and is often heralded by defervescence, improvement of leukopenia, and the occurrence of severe thrombocytopenia, with platelet counts dropping drastically below $100 \times 10^{9} / \mathrm{L}$. Nonetheless, low platelet counts often do not result in overt hemorrhage. It is capillary leakage that gives rise to serious 
complications, and its occurrence marks the onset of the critical phase of dengue, which lasts for approximately 24-48 hours. During this period, clinically or radiologically demonstrable third space fluid accumulation occurs (pleural effusions, ascites), with evidence of hemoconcentration (rising hematocrit and hemoglobin); clinical shock may occur, and, in extreme cases, refractory shock can result in death. Myocarditis, acute respiratory distress syndrome, hepatitis, acute kidney injury, and multi-organ failure can occur. ${ }^{28}$ Although commonly labeled dengue "hemorrhagic" fever, hemorrhage is actually uncommon, and spontaneous hemorrhage usually occurs when the platelet count drops below $5 \times 10^{9} / \mathrm{L}$. However, internal hemorrhage has been reported with higher platelet counts, and is evidenced by shock associated with a drop in hematocrit and hemoglobin. ${ }^{29}$ In extreme cases of dengue, clinical progression can be relentless and unresponsive to treatment, resulting in severe multi-organ failure and death. ${ }^{29}$ A myriad of rare, unusual manifestations have also been reported with dengue, such as encephalitis, Guillain-Barré syndrome, cerebellitis, hemolytic uremic syndrome, rhabdomyolysis, parotitis, acute pancreatitis, acalculous cholecystitis, appendicitis, and others. ${ }^{30}$

Diagnosis of dengue is based on standard diagnostic criteria. In resource-poor settings, clinical criteria are often adequate for making a presumptive diagnosis. Traditionally, confirmation was by detecting dengue immunoglobulin $\mathrm{M}$ enzyme-linked immunosorbent assay. The last 5 years have seen major advances in diagnostics, based on the detection of nonstructural protein- $1,{ }^{31}$ which is secreted by virus-infected cells; of the commonly available diagnostic methods, a combination of nonstructural protein-1 antigen detection and immunoglobulin $\mathrm{M}$ enzyme-linked immunosorbent assay provides the best accuracy. ${ }^{32}$

\section{Management}

Careful monitoring and fluid balance, with emphasis on preventing overhydration, is the mainstay of management. ${ }^{28}$ Previously, mortalities were seen either with over-zealous administration of intravenous fluids or due to inadequate fluid resuscitation; current guidelines ${ }^{5}$ advocate a more conservative approach to fluid administration, favoring oral fluids over intravenous fluids if the patient can drink, and careful titration of intake based on urine output and hemodynamic parameters. Crystalloids and colloids have been shown to have similar effects, with no advantage shown with colloid administration. ${ }^{33-35}$ No specific treatment exists; no antiviral treatment is of benefit, and there are no data to support the use of immunoglobulins. ${ }^{36}$ Despite being contrary to guidelines, some clinicians administer corticosteroids at various stages of the illness. ${ }^{37,38}$ No vaccine is available, and prevention is based largely on public health measures to eradicate mosquito breeding sites.

Despite the lack of convincing evidence of its benefit, trials of corticosteroids in dengue continue, on the basis that ameliorating an overactive immune response may prevent or treat severe dengue where profound vascular leakage occurs. There is little evidence currently on the effects of corticosteroids on the deranged immunological mechanisms occurring in dengue.

\section{Evidence from trials: efficacy, safety}

The earliest evidence of possible benefit of corticosteroids in dengue came from a small randomized controlled trial, in which children with dengue shock syndrome were treated with a tapering dose of hydrocortisone for 3 days; a statistically significant mortality benefit was seen in older children ( 8 years and over). ${ }^{39}$ Several clinical trials were conducted between 1973 and 1988, with varying results (Table 1). ${ }^{40-44}$ In another nonrandomized, non-blinded case series of 22 children with dengue shock syndrome, nine out of eleven patients treated with methylprednisolone survived, while all patients not given steroids died. ${ }^{40}$ Nonetheless, many subsequent controlled studies of corticosteroids in dengue shock syndrome failed to show benefit, ${ }^{41-45}$ and a Cochrane Review concluded that there was no benefit in treatment with corticosteroids in dengue. ${ }^{46}$ The methodological quality and validity of these studies were poor, with high risk of bias and inadequate power; the total pooled number of patients was 284 , and all were children. ${ }^{47}$

Corticosteroids are not recommended treatment in the WHO dengue guidelines. ${ }^{5}$ The main criticism of this recommendation is that it is based on poor-quality evidence, thus the interest in corticosteroids for treating dengue has continued. ${ }^{47}$ A recent retrospective study of single-dose methylprednisolone in adult patients with dengue shock, comparing them with a group of similar patients who did not receive steroids as per protocol, showed a significant reduction in mortality with corticosteroid therapy. ${ }^{48}$ Time to defervescence, hematological recovery, and hospital stay were all significantly shorter in the corticosteroid cohort, and the amount of resuscitation fluids and need for intensive care were considerably less. Nonetheless, this was not a randomized trial, although the results were striking. Notably, this study recruited the sickest of dengue patients, and administration of corticosteroids was a rescue measure; this raises the question as to the critical importance of timing of corticosteroid administration. 


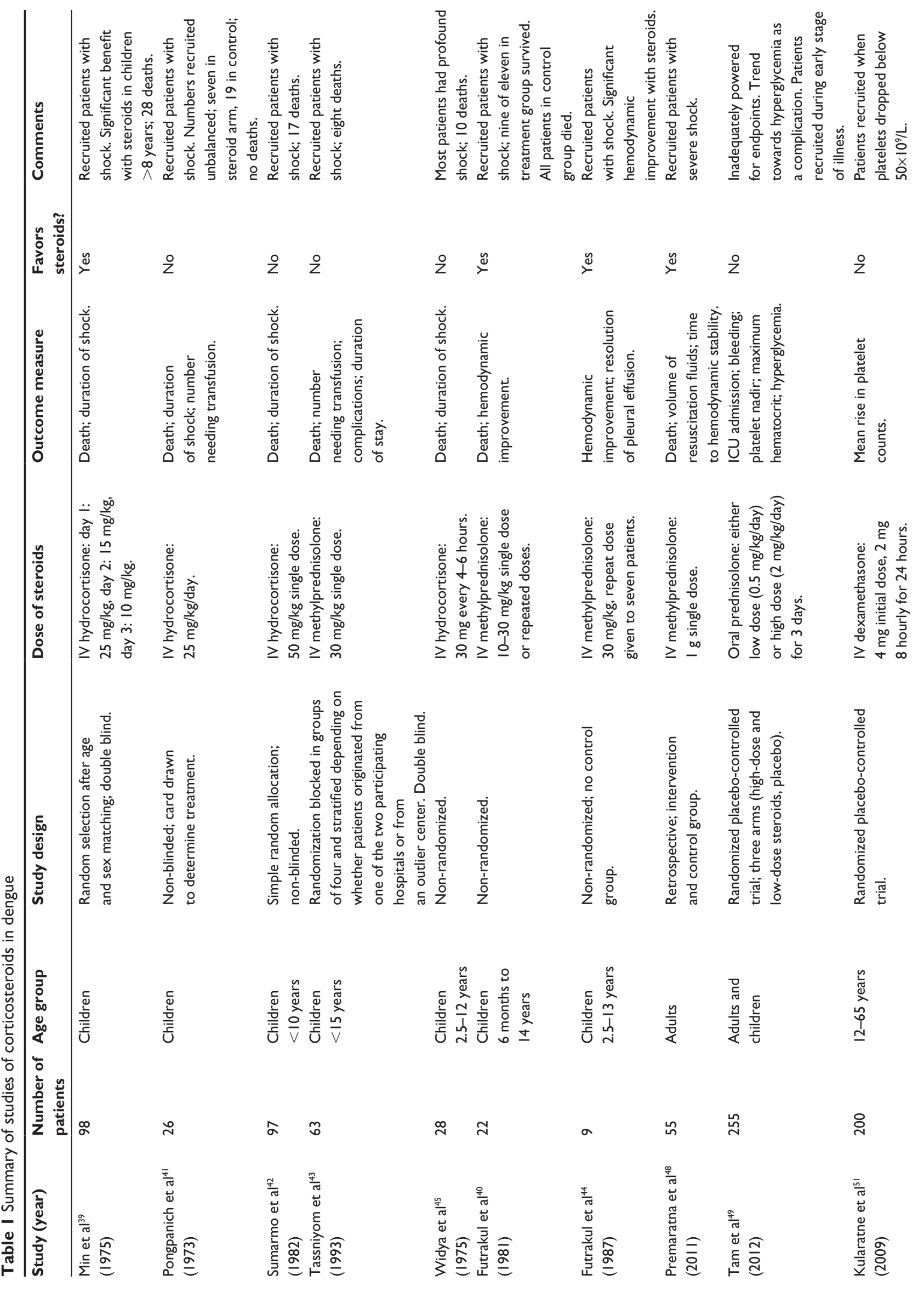




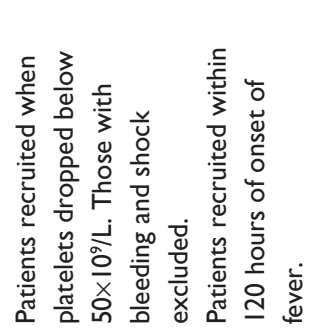

$\stackrel{0}{z}$

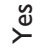

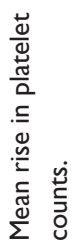

Two studies have evaluated the effects of corticosteroids given early on in the course of dengue illness, prior to the onset of the critical phase. In a relatively large randomized controlled trial in Vietnam comparing high-dose $(2 \mathrm{mg} / \mathrm{kg})$ and low-dose $(0.5 \mathrm{mg} / \mathrm{kg})$ prednisolone for 3 days in the early stages of dengue, no reduction in the incidence of shock or other complications was seen with corticosteroids. ${ }^{49}$ Conversely, another study, published only in abstract form, suggested that administration of high-dose corticosteroids early on in the course of illness, ie, within 120 hours of onset of fever, reduces the incidence of bleeding and ascites. ${ }^{50}$ Corticosteroid treatment was associated with a slightly higher risk of hyperglycemia, but no adverse effects or prolongation of viremia were seen. ${ }^{50}$ There is currently inadequate evidence from which to draw a firm conclusion on the benefits of early administration of corticosteroids.

The studies discussed so far looked at outcome in terms of mortality due to shock, or the prevention of shock and other complications. Corticosteroid treatment has also failed to show improvement in platelet counts in dengue. ${ }^{51,52}$ Some benefit of oral or pulsed methylprednisolone in dengue maculopathy has been suggested, ${ }^{53}$ this may reflect a nonspecific effect of steroids in this situation.

\section{Conclusion}

There is a paucity of high-quality evidence regarding the effects of corticosteroids in dengue, either in prevention of complications or treatment of established shock.

However, the apparent beneficial effects of corticosteroids given for severe shock cannot be ignored, given the high mortality associated with the most severe forms of dengue. There is a definite need for an adequately powered and carefully designed randomized controlled trial of high-dose corticosteroids in the treatment of patients with severe dengue shock syndrome. Justification for such a trial is supported by the fact that no major adverse effects have been demonstrated with the administration of corticosteroids in dengue. The controversy on the effects of corticosteroids in dengue arises from the lack of good-quality trials and the overemphasis of poor-quality evidence from early studies.

\section{Disclosure}

The authors report no conflicts of interest in this work.

\section{References}

1. Weaver SC, Vasilakis N. Molecular evolution of dengue viruses: contributions of phylogenetics to understanding the history and epidemiology of the preeminent arboviral disease. Infect Genet Evol. 2009;9(4):523-540. 
2. Thomas SJ, Strickman D, Vaughn DW. Dengue epidemiology: virus epidemiology, ecology, and emergence. Adv Virus Res. 2003;61: 235-289.

3. Halstead SB. Pathogenesis of dengue: challenges to molecular biology. Science. 1988;239(4839):476-481.

4. Stephenson JR. Understanding dengue pathogenesis: implications for vaccine design. Bull World Health Organ. 2005;83(4):308-314.

5. Dengue: Guidelines for Diagnosis, Treatment, Prevention and Control. Geneva: World Health Organization; 2009. Available from: http:// whqlibdoc.who.int/publications/2009/9789241547871_eng.pdf. Accessed February 14, 2014.

6. Bhatt S, Gething PW, Brady OJ, et al. The global distribution and burden of dengue. Nature. 2013;496(7446):504-507.

7. McBride WJ, Bielefeldt-Ohmann H. Dengue viral infections; pathogenesis and epidemiology. Microbes Infect. 2000;2(9):1041-1050.

8. Malavige GN, Velathanthiri VG, Wijewickrama ES, et al. Patterns of disease among adults hospitalized with dengue infections. QJM. 2006;99(5):299-305.

9. Green S, Vaughn DW, Kalayanarooj S, et al. Early immune activation in acute dengue illness is related to development of plasma leakage and disease severity. J Infect Dis. 1999;179(4):755-762.

10. Halstead SB, O'Rourke EJ. Antibody-enhanced dengue virus infection in primate leukocytes. Nature. 1977;265(5596):739-741.

11. Mongkolsapaya J, Dejnirattisai W, Xu XN, et al. Original antigenic sin and apoptosis in the pathogenesis of dengue hemorrhagic fever. Nat Med. 2003;9(7):921-927.

12. Dejnirattisai W, Jumnainsong A, Onsirisakul N, et al. Cross-reacting antibodies enhance dengue virus infection in humans. Science. 2010;328(5979):745-748.

13. Stephens HA. HLA and other gene associations with dengue disease severity. Curr Top Microbiol Immunol. 2010;338:99-114.

14. Libraty DH, Endy TP, Houng HS, et al. Differing influences of virus burden and immune activation on disease severity in secondary dengue-3 virus infections. J Infect Dis. 2002;185(9):1213-1221.

15. Malavige GN, Ogg GS. T cell responses in dengue viral infections. $J$ Clin Virol. 2013;58(4):605-611.

16. Malavige GN, Ogg G. Pathogenesis of severe dengue infection. Ceylon Med J. 2012;57(3):97-100.

17. Kurane I. Dengue hemorrhagic fever with special emphasis on immunopathogenesis. Comp Immunol Microbiol Infect Dis. 2007; 30(5-6):329-340.

18. Vaughn DW, Green S, Kalayanarooj S, et al. Dengue viremia titer, antibody response pattern, and virus serotype correlate with disease severity. J Infect Dis. 2000;181(1):2-9.

19. Wills BA, Oragui EE, Dung NM, et al. Size and charge characteristics of the protein leak in dengue shock syndrome. J Infect Dis. 2004;190(4): $810-818$.

20. Annane D, Bellissant E, Bollaert PE, et al. Corticosteroids in the treatment of severe sepsis and septic shock in adults: a systematic review. JAMA. 2009;301(22):2362-2375.

21. Dellinger RP, Levy MM, Rhodes A, et al; Surviving Sepsis Campaign Guidelines Committee including the Pediatric Subgroup. Surviving sepsis campaign: international guidelines for management of severe sepsis and septic shock: 2012. Crit Care Med. 2013;41(2):580-637.

22. Annane D, Bellissant E, Bollaert PE, Briegel J, Keh D, Kupfer Y. Corticosteroids for treating severe sepsis and septic shock. Cochrane Database Syst Rev. 2004;(1):CD002243.

23. Annane D. Corticosteroids for severe sepsis: an evidence-based guide for physicians. Ann Intensive Care. 2011;1(1):7.

24. Meduri GU, Headley AS, Golden E, et al. Effect of prolonged methylprednisolone therapy in unresolving acute respiratory distress syndrome: a randomized controlled trial. JAMA. 1998;280(2):159-165.

25. Medin CL, Rothman AL. Cell type-specific mechanisms of interleukin-8 induction by dengue virus and differential response to drug treatment. J Infect Dis. 2006;193(8):1070-1077.

26. Nguyen TH, Nguyen TH, Vu TT, et al. Corticosteroids for dengue - why don't they work? PLoS Negl Trop Dis. 2013;7(12):e2592.
27. Duyen HT, Ngoc TV, Ha do T, et al. Kinetics of plasma viremia and soluble nonstructural protein 1 concentrations in dengue: differential effects according to serotype and immune status. J Infect Dis. 2011;203(9):1292-1300.

28. Rajapakse S, Rodrigo C, Rajapakse A. Treatment of dengue fever. Infect Drug Resist. 2012;5:103-112.

29. Rajapakse S. Dengue shock. J Emerg Trauma Shock. 2011;4(1): 120-127.

30. Gulati S, Maheshwari A. Atypical manifestations of dengue. Trop Med Int Health. 2007;12(9):1087-1095.

31. Bessoff K, Phoutrides E, Delorey M, Acosta LN, Hunsperger E. Utility of a commercial nonstructural protein 1 antigen capture kit as a dengue virus diagnostic tool. Clin Vaccine Immunol. 2010;17(6):949-953.

32. Blacksell SD, Jarman RG, Gibbons RV, et al. Comparison of seven commercial antigen and antibody enzyme-linked immunosorbent assays for detection of acute dengue infection. Clin Vaccine Immunol. 2012;19(5):804-810.

33. Dung NM, Day NP, Tam DT, et al. Fluid replacement in dengue shock syndrome: a randomized, double-blind comparison of four intravenousfluid regimens. Clin Infect Dis. 1999;29(4):787-794.

34. Ngo NT, Cao XT, Kneen R, et al. Acute management of dengue shock syndrome: a randomized double-blind comparison of 4 intravenous fluid regimens in the first hour. Clin Infect Dis. 2001;32(2):204-213.

35. Wills BA, Nguyen MD, Ha TL, et al. Comparison of three fluid solutions for resuscitation in dengue shock syndrome. N Engl J Med. 2005;353(9): $877-889$.

36. Rajapakse $\mathrm{S}$. Intravenous immunoglobulins in the treatment of dengue illness. Trans $R$ Soc Trop Med Hyg. 2009;103(9):867-870.

37. Kularatne S. Survey on the management of dengue infection in Sri Lanka: opinions of physicians and pediatricians. Southeast Asian J Trop Med Public Health. 2005;36(5):1198-1200.

38. Rajapakse $\mathrm{S}$, Ranasinghe $\mathrm{C}$, Rodrigo C. Corticosteroid therapy in dengue infection- opinions of junior doctors. J Glob Infect Dis. 2010;2(2): 199-200.

39. Min M, U T, Aye M, Shwe TN, Swe T. Hydrocortisone in the management of dengue shock syndrome. Southeast Asian J Trop Med Public Health. 1975;6(4):573-579.

40. Futrakul P, Vasanauthana S, Poshyachinda M, Mitrakul C, Cherdboonchart V, Kanthirat V. Pulse therapy in severe form of dengue shock syndrome. J Med Assoc Thai. 1981;64(10):485-491.

41. Pongpanich B, Bhanchet P, Phanichyakarn P, Valyasevi A. Studies on dengue hemorrhagic fever. Clinical study: an evaluation of steroids as a treatment. J Med Assoc Thai. 1973;56(1):6-14.

42. Sumarmo, Talogo W, Asrin A, Isnuhandojo B, Sahudi A. Failure of hydrocortisone to affect outcome in dengue shock syndrome. Pediatrics. 1982;69(1):45-49.

43. Tassniyom S, Vasanawathana S, Chirawatkul A, Rojanasuphot S. Failure of high-dose methylprednisolone in established dengue shock syndrome: a placebo-controlled, double-blind study. Pediatrics. 1993;92(1):111-115.

44. Futrakul P, Poshyachinda M, Mitrakul C, et al. Hemodynamic response to high-dose methyl prednisolone and mannitol in severe dengue-shock patients unresponsive to fluid replacement. Southeast Asian JTrop Med Public Health. 1987;18(3):373-379.

45. Widya MS, Martoatmodjo. Clinical observations on dengue shock syndrome (an evaluation of steroid treatment). Paediatr Indones. 1975;15: 151-160.

46. Panpanich R, Sornchai P, Kanjanaratanakorn K. Corticosteroids for treating dengue shock syndrome. Cochrane Database Syst Rev. 2006;(3):CD003488.

47. Rajapakse $\mathrm{S}$. Corticosteroids in the treatment of dengue illness. Trans $R$ Soc Trop Med Hyg. 2009;103(2):122-126.

48. Premaratna R, Jayasinghe KG, Liyanaarachchi EW, Weerasinghe OM, Pathmeswaran A, de Silva HJ. Effect of a single dose of methyl prednisolone as rescue medication for patients who develop hypotensive dengue shock syndrome during the febrile phase: a retrospective observational study. Int J Infect Dis. 2011;15(6):e433-e434. 
49. Tam DT, Ngoc TV, Tien NT, et al. Effects of short-course oral corticosteroid therapy in early dengue infection in vietnamese patients: a randomized, placebo-controlled trial. Clin Infect Dis. 2012;55(9): 1216-1224.

50. Villar LA, Martinez RA, Diaz FA, Villar JC, Rueda E. Effect of methylprednisolone in preventing dengue complications: a singlecenter randomized placebo-controlled trial. Am JTrop Med Hyg. 2009; 81(5 Suppl 1).
51. Kularatne SA, Walathara C, Mahindawansa SI, et al. Efficacy of low dose dexamethasone in severe thrombocytopenia caused by dengue fever: a placebo controlled study. Postgrad Med J. 2009;85(1008):525-529.

52. Shashidhara KC, Sudharshan KA, Basavana H, Bhograj A. Effect of high dose of steroid on plateletcount in acute stage of dengue fever with thrombocytopenia. J Clin Diagn Res. 2013;7(7):1397-1400.

53. Bacsal KE, Chee SP, Cheng CL, Flores JV. Dengue-associated maculopathy. Arch Ophthalmol. 2007;125(4):501-510.

\section{Publish your work in this journal}

Infection and Drug Resistance is an international, peer-reviewed openaccess journal that focuses on the optimal treatment of infection (bacterial, fungal and viral) and the development and institution of preventive strategies to minimize the development and spread of resistance. The journal is specifically concerned with the epidemiology of antibiotic

\section{Dovepress}

resistance and the mechanisms of resistance development and diffusion in both hospitals and the community. The manuscript management system is completely online and includes a very quick and fair peerreview system, which is all easy to use. Visit http://www.dovepress.com/ testimonials.php to read real quotes from published authors.

Submit your manuscript here: http://www.dovepress.com/infection-and-drug-resistance-journal 